\title{
Modeling and Stability Analysis of DC-DC Buck Converter via Takagi-Sugeno Fuzzy Approach
}

\author{
Kamyar Mehran, Member IEEE, Damian Giaouris, Member IEEE, and Bashar Zahawi, Senior Member IEEE \\ School of Electrical, Electronic and Computer Engineering, \\ Newcastle University, Newcastle upon Tyne, UK \\ E-mail: \{kamyar.mehran@ncl.ac.uk\}
}

\begin{abstract}
The DC-DC voltage-mode controlled buck converter is a nonlinear, nonsmooth system that presents different circuit topologies within one switching cycle. This paper develops a Takagi-Sugeno fuzzy approach for modeling this circuit to capture all the essential nonlinearities that occur in fast time scale. Based on the resulting model, we propose tractable mathematical stability analysis for identifying fast-scale instabilities of the converter, more specifically the edge of bifurcation phenomena, employing nonsmooth Lyapunov functions. The search for the Lyapunov functions are formulated as Linear Matrix Inequalities (LMI) feasibility problem which easily can be solved using upto-date interior point methods.
\end{abstract}

\section{INTRODUCTION}

D C to DC converters have long been considered as an exemplary case for studying nonlinear phenomena like subharmonic oscillations and period-doubling bifurcation leading to chaos, which basically arised from their nonsmooth dynamical nature. Accurate mathematical modeling of this group of systems is of great importance mainly in respect to the analysis of the instabilities that occur in fast time scale which the time scale that the switching in the voltage and current waveforms at clock frequency is observable. Problems of this kind are not easy to handle mathematically, since the resulting models are dynamical systems whose righthand sides are not continuous or not differentiable. A number of methods have been proposed for modeling and analyzing the stability of switching power converters [1]-[3] in order to avoid unwanted nonlinearities and chaotic behavior. In brief, the desired stable period-1 operating mode of the converter is a limit cycle whose period equals the period of the clock used in the PWM voltage-mode controller. Variations in system parameters (for instance supply voltage or load resistance) can cause the converter response to enter into period doubling bifurcations. Such bifurcations may lead to subharmonic oscillations, crises and even chaotic behavior [4].

The most conventional approach for studying these limit cycles is nonlinear discrete modeling [1] which generally captures the essential properties of periodic orbits. In many cases, including the voltage-mode controlled buck converter, the derivation of the discrete map analytically is impossible and one has to seek numerical methods. A powerful numerical technique was proposed by the authors [5], [6] to determine the stability of periodic limit cycle in DC-DC buck converter operating in continuous conduction mode. In that study, applying Filippov's method in combination with Floquet theory makes it possible to directly infer the stability of the orbit resulting from small perturbations.

In searching for an alternative approach for accurate mathematical modeling and the most efficient, most straightforward numerical method for rigorous stability analysis of the converter to automatize the whole procedure, model-based TakagiSugeno (TS) fuzzy system design [7], [8] can be considered as a strong candidate. The behavior of these TS fuzzy models is described by a set of rules on the form

$$
\begin{aligned}
\text { Rule } j: & \operatorname{IF} x_{1} \text { is } F_{1}^{j} \text { AND...AND } x_{q} \text { is } F_{q}^{j} \\
& \text { THEN } \dot{x}=A^{j} x+B^{j} u+a^{j}, j=1, \ldots, l
\end{aligned}
$$

and the dynamics of this system can be described by:

$$
\dot{x}=\sum_{j=1}^{l} w^{j}(x)\left(A^{j} x+B^{j} u+a^{j}\right)
$$

where $w^{j}(x)$ are normalized membership functions of the rule antecedents satisfying $0 \leq w^{j}(x) \leq 1, \sum_{j=1}^{l} w^{j}(\theta)=1$ and $l$ is the number of rules. The stability of these systems is based on the existence of a common quadratic Lyapunov function for all linear subsystems and sufficient conditions for stability analysis based on that Lyapunov function [8], [9]. The finesse of this analysis as a numerical approach comes from the fact that the search for Lyapunov function can be formulated as linear matrix inequalities (LMI's). The optimization problem can then be solved efficiently with less computational effort using a widely available software like MATLAB.

There have been a number of successful applications of TS fuzzy methods in power electronic converters [10]-[13]. In terms of the model-based fuzzy approach that blends fuzzy logic and the theory of modern control, the earlier papers approximated the dynamical model of the converter by TS fuzzy model, which is obtained using averaging technique. While the averaging technique is suitable for deriving some information about the stability and dynamic behavior in slowtime scale, it cannot capture the events that occurs at clock frequency. Therefore, the instabilities that may occur in fasttime scale cannot be taken into account and be studied.

In the following parts of this paper, after presenting the governing equations of the voltage-mode controlled buck converter, section-3 is dedicated to extending the TS fuzzy model method to consider all the possible nonlinear phenomena that take place at fast time scale including subharmonic oscillations, crises and chaotic behavior [4]. This demands the integration of some discrete events with TS local linear models 


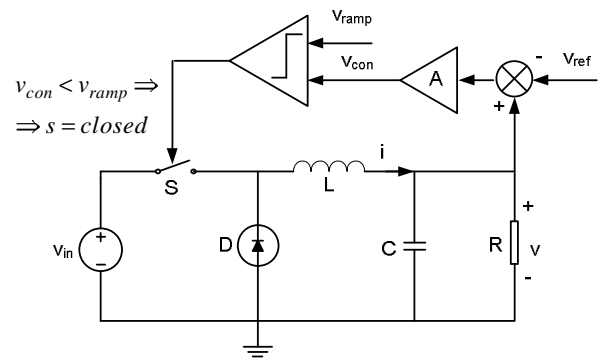

Fig. 1. The voltage mode controlled buck dc-dc converter.

to uniformly represent the nonsmooth dynamical behavior of the converter. Stability conditions are discussed in section4 for the resulting TS model of the converter, based on nonsmooth Lyapunov functions. The analysis can easily point the deviation from period-1 stable operation via the resulting LMI feasibility problem.

\section{THE BUCK CONVERTER AND ITS MATHEMATICAL MODEL}

The voltage mode controlled buck converter circuit shown in Fig. 1 is a nonsmooth dynamical system described by two sets of differential equations:

$$
\begin{gathered}
\frac{d v(t)}{d t}= \begin{cases}\frac{v_{i n}-v(t)}{L}, & \mathrm{~S} \text { is blocking } \\
-\frac{v(t)}{L}, & \mathrm{~S} \text { is conducting }\end{cases} \\
\frac{d v(t)}{d t}=\frac{i(t)-\frac{v(t)}{R}}{C}
\end{gathered}
$$

Switch $S$, which is controlled by pulse-width modulated signal, creates a discontinuity in the right hand side of (2). In the voltage-mode control scheme, the output voltage is compared with the reference voltage to generate a control voltage signal $v_{c o n}=A\left(v(t)-V_{\text {ref }}\right)$ where $A$ is a feedback amplifier gain. The error voltage is then compared with a periodic sawtooth signal $v_{\text {ramp }}$ to generate the switching signal: if $v_{\text {con }}<v_{\text {ramp }}$ then $S$ is conducting and $v_{\text {con }}>v_{\text {ramp }}$ then $S$ is blocking.

Normally, the output of the converter will be a dc voltage with a periodic ripple, with a mean value close to the desired voltage and a period that is equal to the period of the PWM ramp signal (referred to as period-1 waveform), as shown in Fig. 2. In Fig. 3, the same stable period-1 orbit is illustrated in $v-i$ space where after the 9 th time point the time-varying switching surface return to the so-called the fixed point of the cycle with Poincaré map $X(0)$ [1], repeating the periodic cycle.. It has been shown that if a system parameter (say, the input voltage) is varied, the circuit may lose stability through successive period-doubling bifurcation leading to chaos as apparent in Fig. 4 [3], [4].

If we define $x_{1}(t)=v(t)$ and $x_{2}(t)=i(t)$, the equations (2) and (3) can be written as:

$$
\dot{x}= \begin{cases}A_{s} x+B u, & \left(A x_{1}(t)-V_{r e f}\right)<v_{\text {ramp }}(t), \\ A_{s} x, & \left(A x_{1}(t)-V_{\text {ref }}\right)>v_{\text {ramp }}(t) .\end{cases}
$$

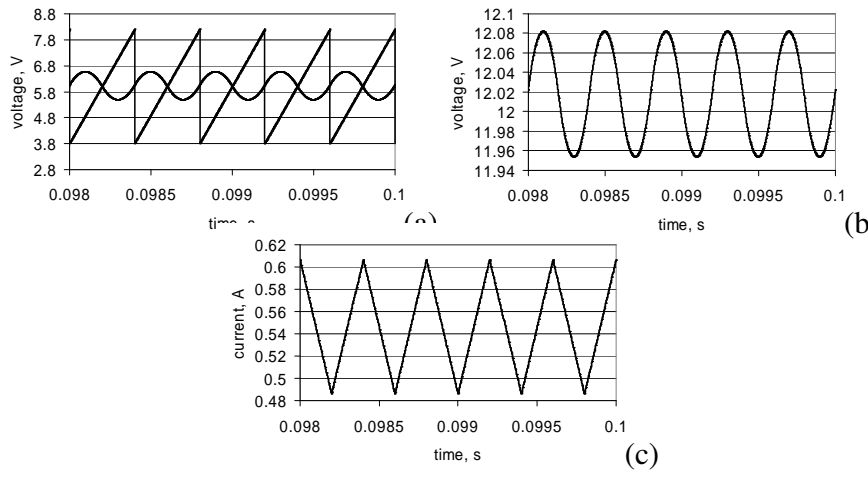

Fig. 2. The nominal period-1 operation of the buck converter: (a) the control and ramp signals, (b) the output voltage, and (c) the output current. The parameter values are $V_{\text {in }}=24 V, V_{\text {ref }}=11.3 \mathrm{~V}, L=20 \mathrm{mH}, R=22 \Omega$, $C=47 \mu F, A=8.4, T=1 / 2500$ s, the ramp signal varies from $3.8 \mathrm{~V}$ to $8.2 \mathrm{~V}$.

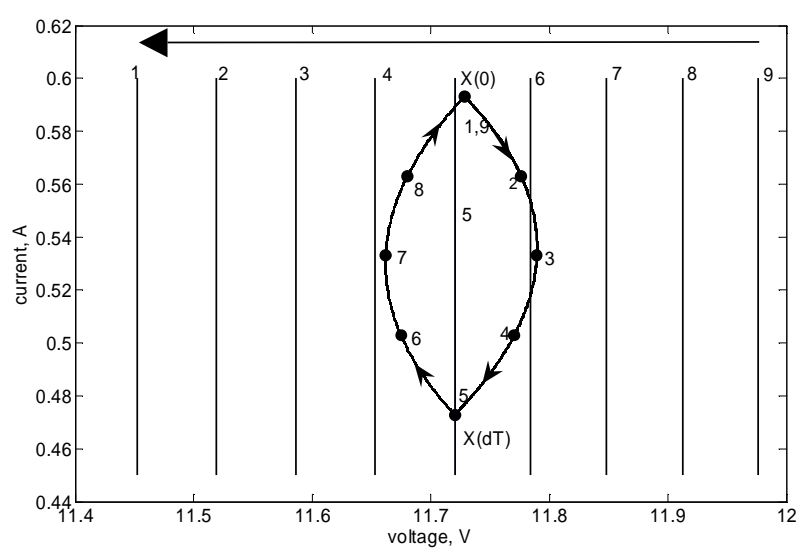

Fig. 3. Period 1 limit cycle, the 9 different points indicate the location of the time varying switching surface at theses instants.

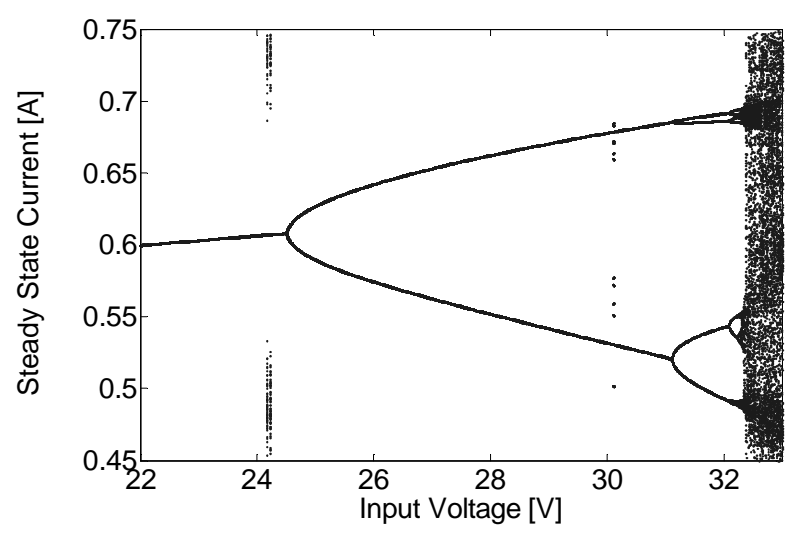

Fig. 4. Bifurcation diagram of the buck converter varying input voltage as a parameter. 
where we can define the state matrices:

$$
A_{s}=\left[\begin{array}{cc}
-1 / R C & 1 / C \\
-1 / L & 0
\end{array}\right], B=\left[\begin{array}{c}
0 \\
1 / L
\end{array}\right] V_{\mathrm{in}}
$$

The switching hypersurface $(h)$ can be written as:

$$
h(x(t), t)=x_{1}(t)-V_{\text {ref }}-\frac{v_{\text {ramp }}}{A}=0, A \neq 0 .
$$

where the periodic sawtooth waveform is defined as:

$$
v_{\text {ramp }}(t)=V_{L}+\left(V_{U}-V_{L}\right)\left(\frac{t}{T} \bmod 1\right)
$$

In Fig. (3), the period-1 orbit is shown in $v-i$ space with 9 different time points. After the 9th point the time-varying switching surface return to the so-called the fixed point of the cycle with Poincaré map $X(0)$ [1], repeating the periodic cycle.

\section{TS FUZZY MODEL OF THE BUCK CONVERTER FOR FAST-SCALE ANALYSIS}

Universal approximation capability of the fuzzy models of the form (1) is discussed in [8]. It has been shown that the affine structure originally proposed in [7] and later in many other applications can approximate any smooth nonlinear function to arbitrary accuracy. However, the function approximation capability of the fuzzy models of the form (1) is fundamentally inadequate to represent the discontinuous dynamics and the ensuing nonlinear events in the example buck converter. To empower the TS fuzzy modeling approach to mathematically represent any switching events, we need to introduce discrete states to interpolate with their associated continuous states. Moreover, the conventional TS fuzzy model can only approximate the functions satisfying local Lipschitz conditions for any interval. Nevertheless, the mathematical model of the buck converter (2), (3) does not fulfil this property at the point of discontinuity according to the definition of the Lipschitz condition [14]. For this reason we need an extra element (discrete events) to hold the existence (and uniqueness) of the fuzzy approximation representing nonsmooth functions [14].

To overcome the shortcomings stated above, a novel TS fuzzy modeling approach is synthesized and presented here to enable modeling of the nonsmooth dynamical equations of the buck converter. The behavior of these models can be described by:

$$
\begin{aligned}
\text { Rule } j & : \operatorname{IF} x_{1} \text { is } F^{j} \\
\text { THEN } \dot{x} & =\left\{\begin{array}{l}
A^{j}\left(m_{i}\right) x+B^{j}\left(m_{i}\right) u+a^{j}\left(m_{i}\right) \\
m^{+}=\varphi(x, m), \quad j=1,2, \quad i=1,2
\end{array}\right.
\end{aligned}
$$

and by the appropriately restricting the inference parameters, the dynamics of the discontinuous fuzzy system can be described by:

$$
\left\{\begin{array}{l}
\dot{x}=\sum_{j=1}^{l} w^{j}\left(x, m_{i}\right)\left(A^{j}\left(m_{i}\right) x+B^{j}\left(m_{i}\right) u+a^{j}\left(m_{i}\right)\right) \\
m^{+}=\phi(x, m)
\end{array}\right.
$$

where $x \in R^{n}$ is the continuous state, $m \in M=\left\{m_{1}, m_{2}\right\}$ is the discrete state, $A^{j}\left(m_{i}\right) \in \Re^{n \times n}, B^{j}\left(m_{i}\right) \in \Re^{n}$, $w^{j}: \Re^{n} \times M \rightarrow\left[\begin{array}{ll}0 & 1\end{array}\right], j \in I_{l}$, are continuous weighting functions which satisfy $\sum_{j=1}^{l} w^{j}(x, m)=1, l$ is the number of fuzzy rules and $F^{j}$ are fuzzy sets. The state space is the Cartesian product $\Re^{n} \times M$. The function $\phi: \Re^{n} \times M \rightarrow M$ describes the dynamics of the discrete state. The notation $m^{+}$ means the next state of $m$. Any value of discrete state $m_{i} \in M$ is associated with an affine subsystem like:

if $\forall x \in A\left(m_{i}\right) x+B\left(m_{i}\right)+a\left(m_{i}\right)$ then $m_{i} \in M, i \in\{1,2\}$

Remark 1: In general a value of $m_{i}$ could be associated with a subset of subsystem as:

if $\forall x \in\left\{\sum_{j \in\{1,2, \ldots\}} w^{j}\left(x, m_{i}\right)\left(A^{j}\left(m_{i}\right) x+B^{j}\left(m_{i}\right) u+\right.\right.$ $\left.\left.a^{j}\left(m_{i}\right)\right)\right\}$ then $m_{i} \in\left\{m_{1}, m_{2}, \ldots, m_{N}\right\}$ when $N$ is possibly infinite

The transition from one discrete state to another means the abrupt change from one set of fuzzy subsystems representing a continuous vector field to another set, which is formally described by the function $\phi$. For convenience, this transition can be defined by a set of switch sets which in fact represent the hypersurface (6). So a switch set can in general be described as:

$$
S_{i, k}=\left\{x \in R^{n} \mid m_{k}=\phi\left(x, m_{i}\right)\right\}, m_{i} \neq m_{k}, i, k \in I_{N}
$$

and, referring to the hypersurface equation (6), the switch set can be defined as:

$$
\begin{gathered}
S_{1,2}=\left\{x \in R^{n} \mid x_{1}(d T)-V_{\text {ref }}<\frac{v_{\mathrm{ramp}}}{v_{\mathrm{ramp}}^{A}}\right\}, \\
S_{2,1}=\left\{x \in R^{n} \mid x_{1}(d T)-V_{\mathrm{ref}}>\frac{v}{A}\right.
\end{gathered}
$$

where $d$ is the duty ratio at each instant. Now we define two membership functions to exactly represent each vector field of the buck converter as follows:

$F^{1}\left(x_{1}(t)\right)=\frac{1}{2}+\frac{x_{1}(t)-X_{1}(0)}{22.6}, F^{2}\left(x_{1}(t)\right)=1-F^{1}\left(x_{1}(t)\right)$

where the state $X(0)=[12.0747,0.6220]^{T}$ is the stable fixed point of the system, which is an intersection point of limit cycle with the poincaré map (Fig. 6) (see [1], [5], [6] for the detailed derivation using Newton-Raphson method). The main reason for selecting the fixed point for constructing the membership functions is to minimize the error of the fuzzy approximation at the switching instants. The shape of fuzzy membership function illustrated in Fig. 5 is selected to exactly model each vector field (2) and (3) as a smooth function. Further detail and examples on defining the fuzzy set in exact linearization method of smooth nonlinear functions is discussed in [8].

The fuzzy model matrices are constructed directly using (5) as $A^{1}\left(m_{1}\right)=A^{2}\left(m_{1}\right)=A^{1}\left(m_{2}\right)=A^{2}\left(m_{2}\right)=A_{s}$, $B\left(m_{1}\right)=B$ and $B\left(m_{2}\right)=\left[\begin{array}{ll}0 & 0\end{array}\right]^{T}$. The discrete state $m_{1}$ is associated with the switch-off vector field and $m_{2}$ is associated with the switch-on vector field of the converter.

To verify the accuracy of how the new modeling approach is able to represent the fast-scale nonlinearities of the system, the time response of the TS fuzzy model of the converter and the original system (Fig. 1) is compared under voltage mode control. Figures 6, shows how the TS fuzzy model exactly mimics the behavior of the original system both in stable 


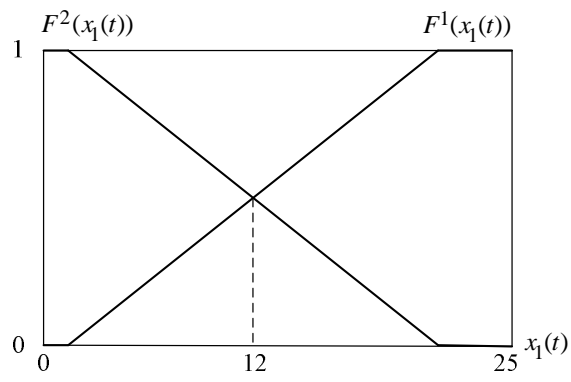

Fig. 5. The trapezoidal membership functions of the TS fuzzy model of buck converter.
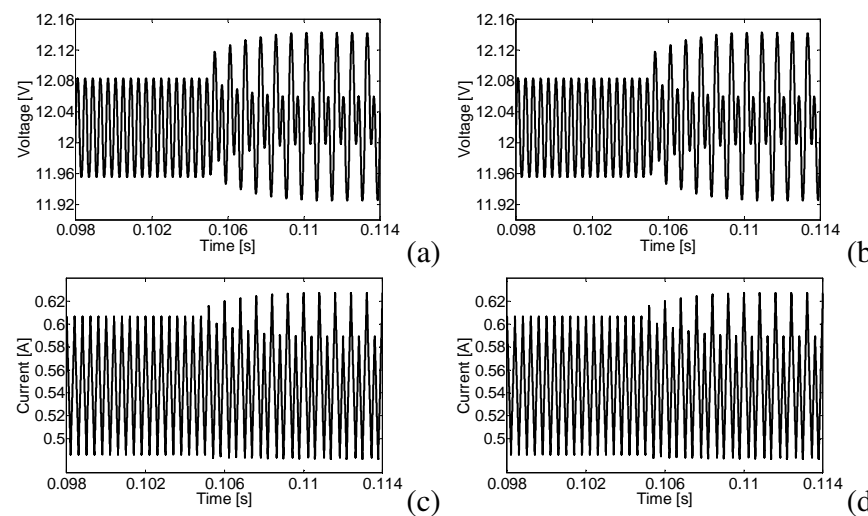

Fig. 6. System output voltage and current time response shows period-1 behavior which is gone to unstable period- 2 at time 0.105 with, (a) original model (b) TS fuzzy model. Likewise the current time responses with (c) original model (d) TS fuzzy model.

period-1 and when the system loses its stability to period doubling bifurcation by changing the input voltage from $24 \mathrm{~V}$ to $25 \mathrm{~V}$. The bifurcation diagram produced using the new model (Fig. 7) also shows the same successive subharmonics and chaotic region comparing to the original system (Fig. 4).

\section{EXPONENTIAL STABILITY ANALYSIS}

The exponential stability of a linear TS fuzzy systems approximating a smooth function [8] is usually stated as follows:

Proposition: The system $\dot{x}=\sum_{j=1}^{l} w^{j}(\theta) A^{j} x$ is exponentially stable if there exist a matrix $P=P^{\mathrm{T}}$ such that

$$
\left\{\begin{array}{l}
P>0 \\
\left(A^{j}\right)^{T} P+P A^{j}<0, \quad \forall j=1,2, \ldots, l
\end{array}\right.
$$

with considering the smooth quadratic Lyapunov function candidate $V(x)=x^{\mathrm{T}} P x$. The stability analysis of affine TS fuzzy systems as described by (1) obtained from smooth functions is also thoroughly discussed in [15].

Considering the fact that the proposed TS fuzzy model of the converter represents a nonsmooth dynamical system, the LMI formulation above for finding global quadratic Lyapunov function in entire fuzzy state space is very conservative. Even in the case of TS fuzzy model of smooth dynamical systems, a global smooth quadratic Lyapunov function fails to exist while the system is actually stable [15], [16]. Hence, very few efficient methods are available (if there are applicable)

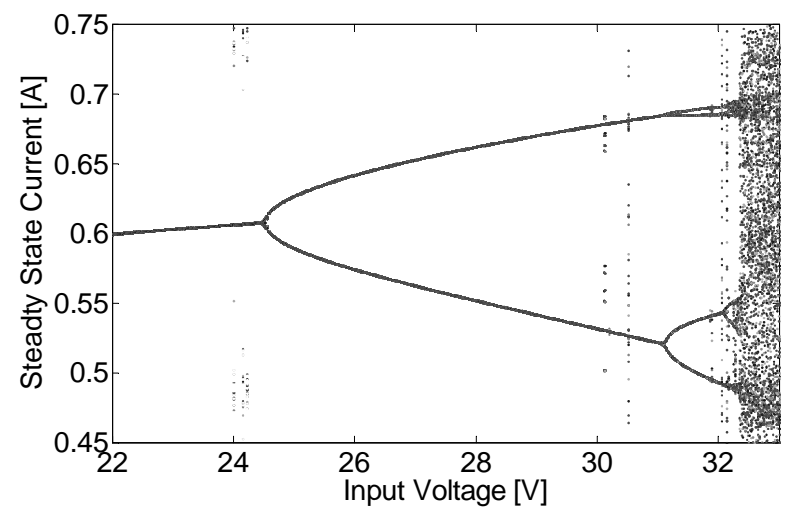

Fig. 7. Bifurcation diagram of the TS fuzzy model of buck converter varying input voltage as a parameter.

to formulate LMI stability conditions based on smooth Lyapunov function. To overcome this conservative formulation for the stability analysis of nonsmooth TS fuzzy model of the buck converter, two natural extension are applied. First, the Lyapunov function candidate is selected as discontinuous or piecewise smooth functions. Second, the fuzzy state space can be partitioned to different flexible regions for the system of the form (9).

The method described in this section can be applied to the proposed TS fuzzy model of the buck converter, and is based on formulating the stability condition as LMIs.

Assume that the fuzzy state space is partitioned into $\Delta$ detached regions $\Omega_{q}, q \in I_{\Delta}$ where $I_{\Delta}=\{1, \ldots, \Delta\}$. The candidate Lyapunov function will be piecewise quadratic, meaning that each local Lyapunov function has the structure:

$$
V(x)=V_{q}(x)=\tilde{x}^{T} \tilde{P}_{q} \tilde{x} \text { when }(x, m) \in \Omega_{q}
$$

where $\tilde{x}=\left[\begin{array}{l}x \\ 1\end{array}\right], \tilde{P}_{q}=\left[\begin{array}{cc}P_{q} & p_{q} \\ p_{q}^{T} & \pi_{q}\end{array}\right], \pi_{q} \in \Re, p_{q} \in \Re^{n}, P_{q}=$ $P_{q}^{T} \in \Re^{n} \times \Re^{n}$ and $q \in I_{\Delta}$.

Let $\Omega_{q}^{x}$ denote the continuous state of $x$ in $\Omega_{q} . V_{q}: \mathbf{c l} \Omega_{q}^{x} \rightarrow$ $\Re, q \in I_{\Delta}$, is a (scalar) function which is assumed to be continuously differentiable on closure of region $\Omega_{q}$ (cl. denotes the closure of a set, which is the smallest closed set containing the set). In fact, the scalar function $V_{q}(x, t)$ is used to measure the fuzzy system's energy in a local region $\Omega_{q}$.

For the sake of relaxing the conservative formulation of stability conditions, the fuzzy state space is partitioned into $\Delta$ detached regions $\Omega_{q}, q \in I_{\Delta}$. A trajectory initiated in any region at time $t_{k}, k=1,2, \ldots$ can pass through another region if $t_{k}<t_{k+1}$. We define $\Lambda_{q r}$ as a neighboring region which means:

$$
\Lambda_{q r}=\left\{x \in \Re^{n} \mid \exists t<t_{0}, \text { such that } x\left(t^{-}\right) \in \Omega_{q}, x(t) \in \Omega_{r}\right\}
$$

$\Lambda_{q r}$ is given by the hypersurface of the form (6). Therefore, if $\Lambda_{q r} \neq \emptyset, \Omega_{q}$ and $\Omega_{r}$ must be neighboring sets. As a sufficient condition let:

$$
\mathrm{I}_{\Lambda}=\left\{(q, r) \mid \Lambda_{q r} \neq \emptyset\right\}
$$

which is a set of tuples indicating that there is at least one point for which the trajectory passes from $\Omega_{q}$ to $\Omega_{r}$. 
Considering the fuzzy region portioning above, (13) is a discontinuous Lyapunov function at the neighboring regions $\Lambda_{q r},(q, r) \in I_{\Lambda}$. Assuming $t_{k}<t_{k+1}$ for every trajectory with initial point in any region, $V(x)$ is piecewise continuous function with respect to time.

\section{Stability analysis as LMI}

Stability conditions presented in this section are supposed to be confined to a part of the continuous state space. It is possible by expressing the region as positive (quadratic) functions and employing a so-called S-procedure technique [17], to substitute the confined conditions with unconfined conditions. This procedure is indispensable to recast all of the conditions on LMIs, so this is initially described in general terms and then the stability theorem is formulated with confined conditions in one LMI feasibility problem.

Let $Q_{0}, \ldots, Q_{s}$, be quadratic functions of the variable $x \in$ $\Re^{n}$ on the form:

$$
Q_{k}(x)=x^{T} Z_{k} x+2 c_{k}^{T}+d_{k}, k=0, \ldots, s,
$$

where $Z_{k}=Z_{k}^{T}$. We consider the following condition on $Q_{0}$ :

$$
Q_{0}(x) \geq 0 \text { in the region }\left\{x \in \Re^{n} \mid F_{k}(x) \geq 0, k \in I_{s}\right\}
$$

The confined condition (17) can be substituted by an unconfined condition in the following way:

Lemma [17]: if there exist $\delta_{k} \geq 0, k \in I_{s}$, such that

$$
\forall x \in \Re^{n}, Q_{0}(x) \geq \sum_{k=1}^{s} \delta_{k} Q_{k}(x)
$$

then (17) holds. Hence, by introducing additional variables $\delta_{k} \geq 0, k \in I_{s}$, condition (17) can be turned into an LMI which can be written as:

$$
x^{T}\left[\begin{array}{cc}
Z_{0} & c_{0} \\
c_{0}^{T} & d_{0}
\end{array}\right] x \geq \sum_{k=1}^{s} \delta_{k} x^{T}\left[\begin{array}{cc}
Z_{0} & c_{0} \\
c_{0}^{T} & d_{0}
\end{array}\right] x
$$

The replacement of (17) by Lemma may be conservative. However it can be shown that the converse is true in case of single quadratic form, $s=1$ [17] contingent on the existence of some $x$ such that $Q_{1}(x)>0$. In case of hypersurface which can be defined by $Q_{k}(x)=0, k \in I_{s}$, Lemma is true without the restriction $\delta_{k} \geq 0$.

Now, by defining $\tilde{I}=\left[\begin{array}{ll}I & 0 \\ 0 & 0\end{array}\right]$ where $\tilde{I} \in \Re^{n+1} \times \Re^{n+1}$, $\tilde{A}\left(m_{i}\right)=\left[\begin{array}{cc}A\left(m_{i}\right) & B\left(m_{i}\right) \\ 0 & 0\end{array}\right], m_{i} \in M$ and applying the above procedure the stability conditions can be stated. All conditions in the stability theorem are described by $Q_{0}(x) \geq 0$, where $Q_{0}(x)$ is a quadratic function defined by (16). The first condition is defined by two inequalities $Q_{0}(x)=\tilde{x}^{T}\left(\tilde{P}_{q}-\alpha \tilde{I}\right) \tilde{x} \geq 0$ and $Q_{0}(x)=\tilde{x}^{T}\left(\beta \tilde{I}-\tilde{P}_{q}\right) \tilde{x} \geq 0$ where $\alpha$ and $\beta$ are constants which originally represent class $\mathcal{K}$ function $\alpha: \Re^{+} \rightarrow \Re^{+}$ and $\beta: \Re^{+} \rightarrow \Re^{+}$of $\|x\|$ [14]. The second condition is $Q_{0}(x)=-\tilde{x}^{T}\left(\tilde{A}(m)^{T} \tilde{P}_{q}+\tilde{P}_{q} \tilde{A}(m)+\gamma \tilde{I}\right) \tilde{x} \geq 0$ where $\gamma>0$ is a scalar constant. The third inequality condition is $Q_{0}(x)=\tilde{x}^{T}\left(\tilde{P}_{r}-\tilde{P}_{q}\right) \tilde{x} \geq 0$. The first and second condition of the stability theorem should be satisfied in regions $\Omega_{q}^{x}$ and $\Omega_{q}^{x, m_{i}}$ respectively. These conditions can be substituted by the unconfined condition (19). The third condition is satisfied on the hypersurface $\Lambda_{q r}^{x}$ which can be given by $Q_{k}(x)=0, k \in I_{s}$, where each $Q_{k}(x)=0$ has the form (16) with no limitation on $\delta_{k}$ as mentioned before.

LMI problem: If there exist $\tilde{P}_{q}, q \in I_{\Delta}$, constants $\alpha>0, \mu_{k}^{q} \geq 0, \nu_{k}^{q i j} \geq 0, \eta_{k}^{q r}$ and a solution to $\min \beta$ subject to the three conditions:

$$
\begin{aligned}
& -\alpha \tilde{I}+\sum_{k=1}^{s_{q}} \mu_{k}^{q}\left[\begin{array}{cc}
Z_{k}^{q} & c_{k}^{q} \\
\left(c_{k}^{q}\right)^{T} & d_{k}^{q}
\end{array}\right] \leq \tilde{P}_{q} \\
& \tilde{P}_{q} \leq \beta \tilde{I}+\sum_{k=1}^{s_{q}} \mu_{k}^{q}\left[\begin{array}{cc}
Z_{k}^{q} & c_{k}^{q} \\
\left(c_{k}^{q}\right)^{T} & d_{k}^{q}
\end{array}\right], q \in I_{\Delta} \\
& \text { - } \quad(q, i, j) \in I_{\Omega},\left(\tilde{A}^{j}\right)^{T} \tilde{P}_{q}+\tilde{P}_{q} \tilde{A}^{j} \\
& +\sum_{k=1}^{s_{q i j}} \nu_{k}^{q i j}\left[\begin{array}{cc}
Z_{k}^{q} & c_{k}^{q} \\
\left(c_{k}^{q}\right)^{T} & d_{k}^{q}
\end{array}\right] \leq-\tilde{I}, q \in I_{\Delta} \\
& \text { - } \tilde{P}_{r} \leq \tilde{P}_{q}-\sum_{k=1}^{s_{q r}} \eta_{k}^{q r}\left[\begin{array}{cc}
Z_{k}^{q r} & c_{k}^{q r} \\
\left(c_{k}^{q r}\right)^{T} & d_{k}^{q r}
\end{array}\right],(q, r) \in I_{\Lambda}
\end{aligned}
$$

Then the fixed point 0 is exponentially stable in the sense of Lyapunov ${ }^{1}$.

Remark 2: Without loss of generality, it is assumed that the origin is a fixed point of the fuzzy system (9). For the buck converter, the fixed point mentioned above is the fixed point of limit cycle with a stroboscopic map [5]

In order to verify the analysis presented above, the fuzzy state-space $\mathcal{F}$ is first partitioned into $\Delta=2$ detached regions $\left(I_{\Delta}=\{1,2\}\right)$ :

$$
\begin{aligned}
& \Omega_{1}=\left\{(x, m) \in \mathcal{F} \mid x \in \Re^{n}, m=m_{1}\right\} \\
& \Omega_{2}=\left\{(x, m) \in \mathcal{F} \mid x \in \Re^{n}, m=m_{2}\right\}
\end{aligned}
$$

Solving the LMI problem for the value of supply voltage $V_{\text {in }}$ $=24 \mathrm{~V}$ results in a solution:

$$
\begin{gathered}
\tilde{\mathbf{P}}_{\mathbf{1}}=\left[\begin{array}{ccc}
2.2526 & -12.8865 & -39.1678 \\
-12.8865 & 0.0026 & -103.3283 \\
-39.1678 & -103.3283 & 0.0004
\end{array}\right] \\
\tilde{\mathbf{P}}_{\mathbf{2}}=\left[\begin{array}{ccc}
2.2526 & 12.8865 & -39.1678 \\
12.8865 & 387.3544 & 103.3283 \\
-39.1678 & 103.3283 & 2235.9155
\end{array}\right]
\end{gathered}
$$

with the optimal value of $\beta=2.4962$. Finding the feasible solution to LMI problem as above clearly means that the system is exponentially stable as it is readily perceived from Fig. 7 showing stable period-one response in this operating point. By changing the supply voltage to $V_{\text {in }}=25 \mathrm{~V}$ no feasible solution can be found for the LMI problem, which obviously implies instability in new operating point. The stability analysis via Filippov method also reconfirm the unstable period-1 orbit coexisting with period-2 orbit for $V_{\text {in }}=25 \mathrm{~V}$ [5] and hence

\footnotetext{
${ }^{1}$ The proof of this theorem is out of the scope of this paper and it will present in later publications.
} 
the prowess of new method for fast-scale stability analysis of the converter.

It is worth noting that by neglecting the fuzzy state space partitioning into two regions, no feasible solution can be found for LMI problem while the converter response is actually in a stable period-1 orbit. This indicates the essential role of region partitioning of the fuzzy state-space in stability conditions specifically in the case of nonsmooth system like the DC-DC buck converter.

\section{CONCLUSION}

We have extended Takagi-Sugeno fuzzy modeling approach to represent the discrete switching events of the DC-DC buck converter, which take place in fast time scale and all nonlinear phenomena induced by those switching events.

Based on the resulting TS fuzzy model proposed in this paper, a rigorous mathematical stability analysis is presented to give an insight into typical fast-scale instabilities affiliated with the buck converter. All the stability conditions are formulated as a Linear Matrix Inequality problem, based on the use of piecewise Lyapunov functions to deal with the discontinuous dynamics of the new TS fuzzy representation and to keep away from the conservative formulation of finding a global Lyapunov function that may misleadingly result in infeasible solution. The TS stability analysis successfully predict the emergence of period-doubling bifurcation, which up till now could have only been achieved through complicated discrete nonlinear modeling methods.

The proposed TS modeling and stability analysis approach presented in this paper can also be applied to other types of electronic power converters. Most importantly, the whole modeling and stability analysis approach can be employed as a framework to devise a new type of fuzzy model-based control strategy to suppress unwanted chaos in the buck converter and other related nonsmooth dynamical systems.

\section{REFERENCES}

[1] S. Banerjee and G. Verghese, eds., Nonlinear Phenomena in Power Electronics: attractors, bifurcation, chaos, and nonlinear control. New York, NY 10016-5997: IEEE Press, 1 ed., 2001.

[2] Y. Zhou, C. K. Tse, S. S. Qiu, and F. C. M. Lau, "Applying resonant parametric perturbarion to control chaos in the buck dc/dc converter with phase shift and frequency mismatch considerations," International Journal of Bifurcation and Chaos, vol. 13, pp. 3459-3471, November 2003.

[3] E. Fossas and G. Olivar, "Study of chaos in the buck converter," Circuits and Systems I: Fundamental Theory and Applications, IEEE Transactions on [see also Circuits and Systems I: Regular Papers, IEEE Transactions on], vol. 43, no. 1, pp. 13-25, 1996.

[4] K. Chakrabarty, G. Poddar, and S. Banerjee, "Bifurcation behavior of the buck converter," IEEE Transactions on Power Electronics, vol. 11, no. 3, pp. 439-447, 1996.

[5] D. Giaouris, S. Banerjee, B. Zahawi, and V. Pickert, "Stability analysis of the continuous-conduction-mode buck converter via filippov's method," IEEE Transactions on Circuits and Systems-I, vol. 55, pp. 1084-1096, May 2008.

[6] D. Giaouris, A. Elbkosh, V. Pickert, B. Zahawi, and S. Banerjee, "Control of period doubling bifurcation in dc-dc converters," in International Control Conference (ICC2006), (Glasgow, United Kingdom), 2006.

[7] T. Takagi and M. Sugeno, "Fuzzy identification of systems and its applications to modeling and control," IEEE Transaction on Systems, Man and Cybernetics, vol. 15, no. 1, pp. 116-132, 1985.

[8] K. Tanaka, Fuzzy Control Systems Design and Analysis: a Linear Matrix Inequality Approach. Newark: John Wiley \& Sons, 2001.
[9] L. Wang, Adaptive fuzzy systems and control: design and stability analysis. Prentice-Hall, Inc. Upper Saddle River, NJ, USA, 1994.

[10] Y. Kolokolov, S. Koschinsky, and A. Hamzaoui, "Comparative study of the dynamics and overall performance of boost converter with conventional and fuzzy control in application to PFC," Power Electronics Specialists Conference, 2004. PESC 04. 2004 IEEE 35th Annual, vol. 3, 2004.

[11] W. So, C. Tse, and Y. Lee, "Development of a fuzzy logic controller for DC/DC converters: design, computer simulation, and experimental evaluation," Power Electronics, IEEE Transactions on, vol. 11, no. 1, pp. 24-32, 1996.

[12] K. Lian, J. Liou, and C. Huang, "LMI-based integral fuzzy control of DC-DC converters," IEEE Transactions of Fuzzy Systems, vol. 14, pp. 71-80, February 2006

[13] K. Guesmi, A. Hamzaoui, and J. Zaytoon, "Control of nonlinear phenamena in DC-DC conevrters: Fuzzy logic approach," International Journal of Circuit Theory and Applications, 2008.

[14] H. K. Khalil, Nonlinear Systems. Upper Saddle River, New Jersey: Prentice-Hall, 2nd ed., 1996.

[15] P. Bergsten, Observers and Controllers for Takagi-Sugeno Fuzzy systems. PhD thesis, Örebro, 2001.

[16] M. Johansson, A. Rantzer, and K. Årzén, "Piecewise quadratic stability of fuzzy systems," IEEE Transaction on Fuzzy Systems, vol. 7, no. 6, 1999.

[17] S. Boyd, L. E. Ghaoui, E. Feron, and V. Balakrishnan, Linear Matrix Inequalities in System and Control Theory. Philadelphia: SIAM, 1994. 